Check for updates

Cite this: RSC Adv., 2018, 8, 16937

Received 18th March 2018

Accepted 2nd May 2018

DOI: $10.1039 / \mathrm{c} 8 \mathrm{ra0} 2374 \mathrm{c}$

rsc.li/rsc-advances

\title{
Superlattice-like structure and enhanced ferroelectric properties of intergrowth Aurivillius oxides
}

\author{
He Yang, ${ }^{a}$ Zezhi Chen, ${ }^{a}$ Ranran Peng, (D)*ab Haoliang Huang, (D) ab Zhengping Fu, (D) ab \\ Xiaofang Zhai ${ }^{\mathrm{bc}}$ and Yalin Lu ${ }^{\star a b c}$
}

Aurivillius oxides with an intergrowth structures have been receiving increasing interest because of their special structures and potential outstanding ferroelectric properties. In this work, $\mathrm{Bi}_{3} \mathrm{LaTiNbFeO}_{12}-$ $\mathrm{Bi}_{5} \mathrm{Ti}_{3} \mathrm{FeO}_{15}$ and $\mathrm{Bi}_{3} \mathrm{TiNbO}_{9}-\mathrm{Bi}_{3} \mathrm{LaTiNbFeO}{ }_{12}$ compounds were successfully synthetised using a simple solid-state reaction method. X-Ray diffraction patterns and scanning transmission electron microscopy high angle annular dark field (STEM-HAADF) images confirm the 2-3 and the 3-4 intergrowth structures in $\mathrm{Bi}_{3} \mathrm{TiNbO}_{9}-\mathrm{Bi}_{3} \mathrm{LaTiNbFeO}{ }_{12}$ and $\mathrm{Bi}_{3} \mathrm{LaTiNbFeO}{ }_{12}-\mathrm{Bi}_{5} \mathrm{Ti}_{3} \mathrm{FeO}_{15}$ compounds, respectively. A superlatticelike distortion in these oxides was proposed resulting from the combination of sub-lattices with different $a$ and $b$ parameters, which was validated by XRD refinements and Raman spectra. Polarization-electric field tests and pulsed polarization positive-up negative-down measurements demonstrate that such superlattice-like structures can effectively enhance the intrinsic ferroelectric polarization and coercive field of these oxides, especially when compared with their component oxides $\mathrm{Bi}_{3} \mathrm{TiNbO}_{9}$, $\mathrm{Bi}_{3} \mathrm{LaTiNbFeO}_{12}$ and $\mathrm{Bi}_{5} \mathrm{Ti}_{3} \mathrm{FeO}_{15}$. Simultaneously, ferroelectric Curie temperatures of $\mathrm{Bi}_{3} \mathrm{TiNbO}_{9}-$ $\mathrm{Bi}_{3} \mathrm{LaTiNbFeO}{ }_{12}$ and $\mathrm{Bi}_{3} \mathrm{LaTiNbFeO}{ }_{12}-\mathrm{Bi}_{5} \mathrm{Ti}_{3} \mathrm{FeO}_{15}$ oxides are lowered because of the internal stress in the superlattice-like structure. Nevertheless, the paramagnetism of the samples is hardly influenced by their structure, while mainly related to their iron content, in which iron has a similar effective magnetic moment around 3.4-3.9.

\section{Introduction}

Multiferroic materials exhibiting ferroelectric (FE) and ferromagnetic (FM) properties simultaneously have drawn numerous attentions as a new type of very promising materials which could be widely applied as quantum sensors and storage mediums ${ }^{1-7}$ Among them, Bi-containing Aurivillius compounds with the general formula of $\left(\mathrm{Bi}_{2} \mathrm{O}_{2}\right)^{2+}\left(\mathrm{A}_{n-1} \mathrm{~B}_{n} \mathrm{O}_{3 n+1}\right)^{2-}$ are one of the most important branches. ${ }^{8-12}$ In these Aurivillius compounds, perovskite-like layers $\left(\mathrm{A}_{n-1} \mathrm{~B}_{n} \mathrm{O}_{3 n+1}\right)^{2-}$ are sandwiched by fluorite-like layers $\left(\mathrm{Bi}_{2} \mathrm{O}_{2}\right)^{2+}$, and it's found that the layer number, $n$, has a great impact on their ferroelectric (FE) and ferromagnetic (FM) properties. ${ }^{13,14}$ For example, $\mathrm{Bi}_{7} \mathrm{Fe}_{3} \mathrm{Ti}_{3}$ $\mathrm{O}_{21}(n=6)$ is paramagnetic while $\mathrm{Bi}_{8} \mathrm{Fe}_{4} \mathrm{Ti}_{3} \mathrm{O}_{24}(n=7)$ is more antimagnetic. The remanent polarization $\left(2 P_{\mathrm{r}}\right)$ and coercive

${ }^{a}$ CAS Key Laboratory of Materials for Energy Conversion, Department of Materials Science and Engineering, University of Science and Technology of China, Hefei 230026, P. R. China.E-mail:yllu@ustc.edu.cn; pengrr@ustc.edu.cn

${ }^{b}$ Synergetic Innovation Center of Quantum Information \& Quantum Physics, Hefei National Laboratory for Physical Sciences at Microscale, University of Science and Technology of China, Hefei 230026, P. R. China

${ }^{c}$ National Synchrotron Radiation Laboratory, University of Science and Technology of China, Hefei 230026, P. R. China field $\left(2 E_{\mathrm{c}}\right)$ of $\mathrm{Bi}_{4} \mathrm{SrTi}_{4} \mathrm{O}_{15}(n=4)$ are $15 \mu \mathrm{C} \mathrm{cm}{ }^{-2}$ and $160 \mathrm{kV} \mathrm{cm}^{-1}$ while for $\mathrm{Bi}_{4} \mathrm{Sr}_{2} \mathrm{Ti}_{5} \mathrm{O}_{18}(n=5)$, they are $22 \mu \mathrm{C} \mathrm{cm}{ }^{-2}$ and 90 $\mathrm{kV} \mathrm{cm}{ }^{-1}$ at room temperature. ${ }^{15,16}$ In general, $n$ takes an integer in these single-phase Aurivillius compounds, and very recently, an $n / n+1$ intergrowth structure was observed, which made the average value of $n$ a fraction, such as typical $\mathrm{Bi}_{3} \mathrm{TiNbO}_{9}-$ $\mathrm{Bi}_{4} \mathrm{Ti}_{3} \mathrm{O}_{12}$ with an average $n$ value of 2.5. ${ }^{17,18}$ Such intergrowth structures may be a new regulation approach to intensively modulate both FE and FM properties of these Aurivillius oxides.

In intergrowth Aurivillius oxides, two constituent structures (generally $n$ and $n+1$ ) arrange alternately along the common $c$ axis. As shown in Fig. 1, the intergrowth structure of typical $\mathrm{Bi}_{3} \mathrm{TiNbO}_{9}-\mathrm{Bi}_{4} \mathrm{Ti}_{3} \mathrm{O}_{12}$ oxide consists of the alternating 2-layer $\mathrm{Bi}_{3} \mathrm{TiNbO}_{9}$ and 3-layer $\mathrm{Bi}_{4} \mathrm{Ti}_{3} \mathrm{O}_{12}$, making the average value of layer number $n 2.5 .{ }^{17}$ Similar intergrowth structures have been observed in $\mathrm{Bi}_{2} \mathrm{WO}_{6}-\mathrm{Bi}_{3} \mathrm{TiNbO}_{9}$ (1.5 layers) and $\mathrm{Bi}_{4} \mathrm{Ti}_{3} \mathrm{O}_{12}-\mathrm{Bi}_{5^{-}}$ $\mathrm{Ti}_{3} \mathrm{FeO}_{15}$ (3.5 layers) oxides, and were suggested to have positive effects on their ferroelectric properties. ${ }^{19-21}$ Nevertheless, most recent research studies on these intergrowth oxides are focused on doping various elements to intensively modify their FE/FM properties. ${ }^{22-26}$ Meanwhile the reason why the intergrowth structure can cause such attractive properties and the relationship between the intergrowth structure and their constituent homologous structures have been rarely studied. 
○ $\mathbf{B i}$

- $\mathbf{T i} / \mathbf{N b}$

- $\mathbf{O}$

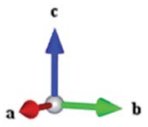

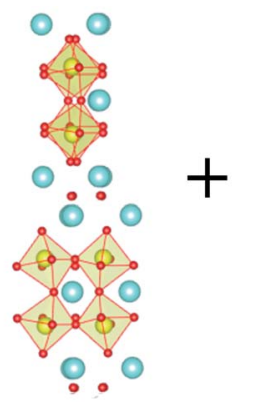
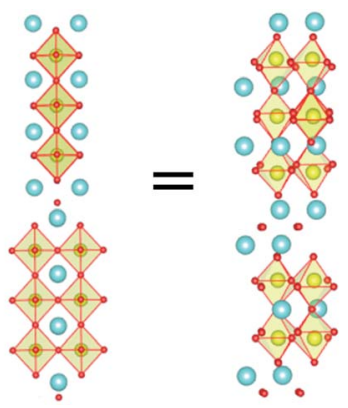

Fig. 1 Schematic representation of the crystalline structure of $\mathrm{Bi}_{3}$ $\mathrm{TiNbO}_{9}-\mathrm{Bi}_{4} \mathrm{Ti}_{3} \mathrm{O}_{12}$.

In this work, new compounds $\mathrm{Bi}_{3} \mathrm{TiNbO}_{9}-\mathrm{Bi}_{3} \mathrm{LaTiNbFeO}_{12}$ and $\mathrm{Bi}_{3} \mathrm{LaTiNbFeO}{ }_{12}-\mathrm{Bi}_{5} \mathrm{Ti}_{3} \mathrm{FeO}_{15}$ with 2.5 and 3.5 layer intergrowth structures were successfully synthesized by the solidstate reaction method. Room temperature X-ray diffraction, STEM-HAADF and Raman spectroscopy were applied to investigate their detailed lattice structure. For comparison, lattice structures of $\mathrm{Bi}_{3} \mathrm{TiNbO}_{9}, \mathrm{Bi}_{3} \mathrm{LaTiNbFeO}_{12}$ and $\mathrm{Bi}_{5} \mathrm{Ti}_{3} \mathrm{FeO}_{15}$ were also studied. Polarization-electric field tests and pulsed polarization positive-up negative-down measurements were used to characterize the ferroelectric and magnetic properties of the samples. Dielectric properties of the samples were measured at

various frequencies and temperatures to determine their Curie temperatures. Magnetic hysteresis loops and the temperature dependence of magnetization for the samples in zero field cooling (ZFC) and field cooling (FC) modes under a magnetic field of 500 Oe were also investigated.

\section{Experimental section}

Powders of $\mathrm{Bi}_{3} \mathrm{LaTiNbFeO}{ }_{12}-\mathrm{Bi}_{5} \mathrm{Ti}_{3} \mathrm{FeO}_{15}$ (BLTNF-3.5) with intergrowth structure were prepared by a conventional solidstate reaction. Appropriate amounts of $\mathrm{Bi}_{2} \mathrm{O}_{3}$ (99.99\%), $\mathrm{TiO}_{2}$ (99.98\%), $\mathrm{Nb}_{2} \mathrm{O}_{5}(99.5 \%), \mathrm{Fe}_{2} \mathrm{O}_{3}(99.9 \%)$ and $\mathrm{La}_{2} \mathrm{O}_{3}(99.98 \%)$ were ball-mixed in agate jars for 24 hours in ethanol. Here $5 \%$ of $\mathrm{Bi}_{2} \mathrm{O}_{3}$ excess was adapted to compensate for the volatilization of bismuth oxide during preparation process. After drying for 24 hours, the milled powders were calcined at $950{ }^{\circ} \mathrm{C}$ for $5 \mathrm{~h}$ to get well crystalline. To get ceramic pellets, BLTNF-3.5 powders were cold-pressed into disks with $12 \mathrm{~mm}$ in diameter and $0.9 \mathrm{~mm}$ in thickness at a pressure of $100 \mathrm{MPa}$, and then sintered at $1150{ }^{\circ} \mathrm{C}$ for $5 \mathrm{~h}$ in air to get dense. Powders and ceramics of $\mathrm{Bi}_{3}$ TiNbO $_{9}$ (BTN-2), $\quad \mathrm{Bi}_{3}$ TiNbO $_{9}-\mathrm{Bi}_{3}$ LaleTiNbFeO $_{12} \quad$ (BLTNF-2.5), $\quad \mathrm{Bi}_{3}$ LaTiNbFeO ${ }_{12}$ (BLTNF-3) and $\mathrm{Bi}_{5} \mathrm{Ti}_{3} \mathrm{FeO}_{15}$ (BTF-4) were also prepared with similar process.

X-Ray Diffraction (XRD) patterns of the synthesized powders were recorded using powder X-ray diffraction (XRD) with $\mathrm{Cu}-\mathrm{K}_{\alpha}$ radiation (TTR-111, Tokyo, Japan). Rietveld refinements of the

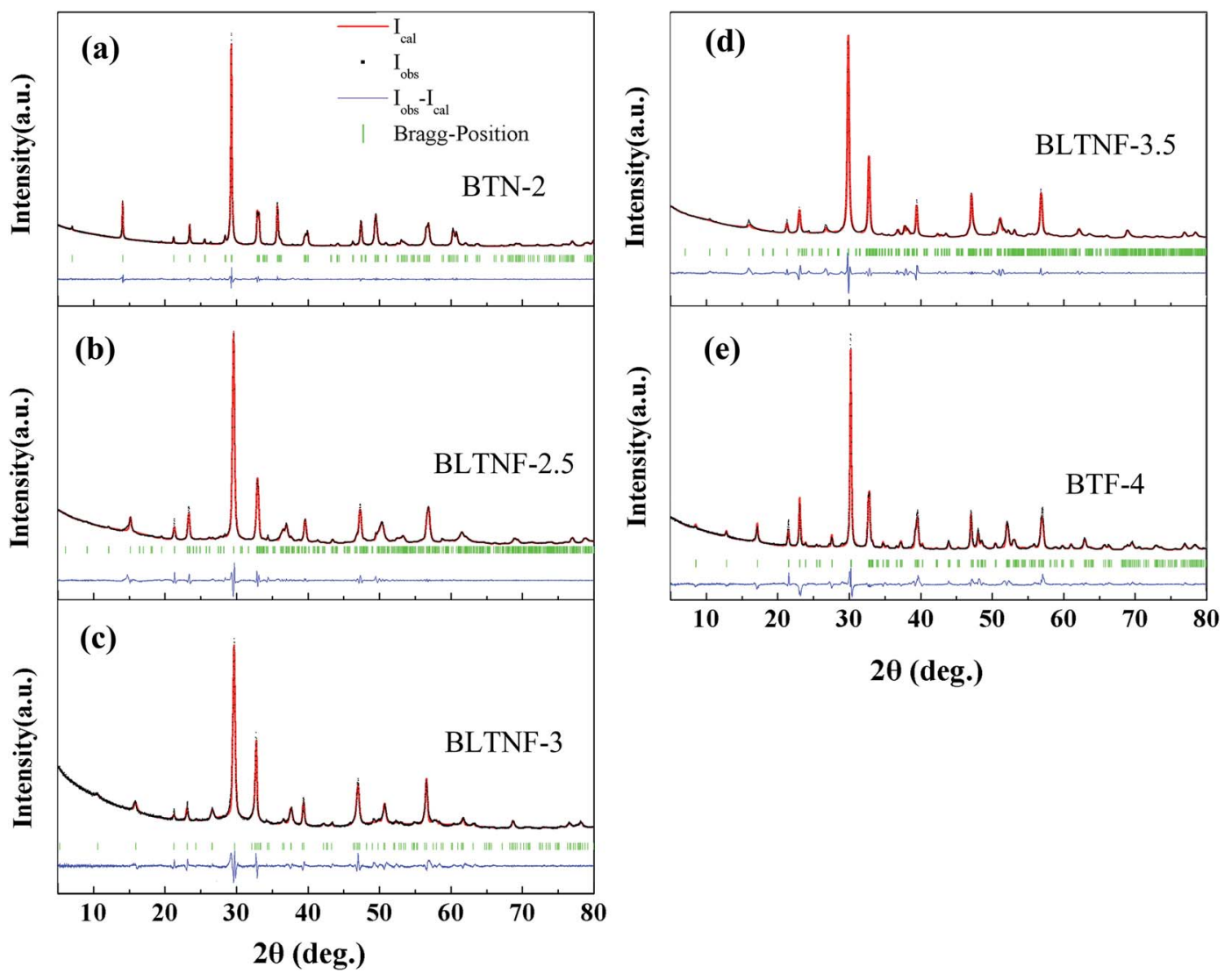

Fig. 2 XRD patterns and refined results of (a) $\mathrm{Bi}_{3} \mathrm{TiNbO}_{9}$ (BTN-2); (b) $\mathrm{Bi}_{3} \mathrm{TiNbO}_{9}-\mathrm{Bi}_{3} \mathrm{LaTiNbFeO}_{12}$ (BLTNF-2.5); (c) Bi $\mathrm{BLaTiNbFeO}_{12}$ (BLTNF-3); (d) $\mathrm{Bi}_{3} \mathrm{LaTiNbFeO}{ }_{12}-\mathrm{Bi}_{5} \mathrm{Ti}_{3} \mathrm{FeO}_{15}$ (BLTNF-3.5); and (e) $\mathrm{Bi}_{5} \mathrm{Ti}_{3} \mathrm{FeO}_{15}$ (BTF-4). 
Table 1 Refined lattice parameters with $R$-factors of the prepared samples

\begin{tabular}{|c|c|c|c|c|c|c|c|}
\hline \multirow[b]{2}{*}{ Compound } & \multicolumn{3}{|c|}{ Lattice parameter $(\AA)$} & \multirow[b]{2}{*}{$R_{\mathrm{wp}}$} & \multirow[b]{2}{*}{$R_{\mathrm{wp}(\mathrm{w} / \mathrm{o} \mathrm{bck})}$} & \multirow[b]{2}{*}{$R_{\mathrm{p}}$} & \multirow[b]{2}{*}{ Space group } \\
\hline & $a$ & $b$ & $c$ & & & & \\
\hline $\mathrm{Bi}_{3} \mathrm{TiNbO}_{9}-\mathrm{Bi}_{3} \mathrm{LaTiNbFeO}_{12}$ & 5.4241 & 5.4678 & 29.1250 & $6.22 \%$ & $12.46 \%$ & $3.73 \%$ & $I 2 \mathrm{~cm}$ \\
\hline $\mathrm{Bi}_{3} \mathrm{LaTiNbFeO}_{12}$ & 5.4675 & 5.5011 & 33.4223 & $5.83 \%$ & $15.69 \%$ & $4.05 \%$ & Fmmm \\
\hline $\mathrm{Bi}_{3} \mathrm{LaTiNbFeO}_{12}-\mathrm{Bi}_{5} \mathrm{Ti}_{3} \mathrm{FeO}_{15}$ & 5.4459 & 5.4896 & 37.3095 & $7.68 \%$ & $17.47 \%$ & $5.36 \%$ & $P 2{ }_{1} a m$ \\
\hline
\end{tabular}

XRD patterns were performed with Material Studio 6.0 program. Atomic structures were visualized by aberration corrected scanning transmission electron microscopy equipped with high angle annular dark field (STEM-HAADF, JEM-ARM200F, JEOL, Japan). Scanning electron microscopy (SEM, JSM-6400; JEOL) was applied to investigate microstructures of the fractured ceramic pellets. Transmission Electron Microscopy (TEM, JEM2100F) and Energy Dispersive Spectroscopy (EDS) were used to determine morphology and elemental composition of BLTNF3.5 powders. Raman spectra were characterized using a Laser Raman spectrometer with $514.5 \mathrm{~nm}$ line of $\mathrm{Ar}^{+}$laser as excitation source (LabRamHr, JobinYvon, France). Magnetic properties of the samples were measured using vibrating sample magnetometer option of Quantum Design Physical Property Measurement System (PPMS-VSM, Quantum Design, San Diego, CA). For ferroelectric and dielectric measurements, ceramics were polished to pellets with $0.2 \mathrm{~mm}$ and $0.5 \mathrm{~mm}$ in thickness, respectively, and then silver was evaporated on both surfaces as electrodes. Ferroelectric measurements were made using a Precision LC ferroelectric analyser at an applied frequency of $50 \mathrm{~Hz}$ (Radiant Technology, Inc., Albuquerque, NM). Dielectric measurements were conducted using an impedance analyser at the frequency range of $5 \mathrm{kHz}-1 \mathrm{MHz}$ (HP4294A, Agilent Technology).

\section{Results and discussion}

Room temperature X-ray diffraction (XRD) patterns of samples, as shown in Fig. 2, indicate the formation of Aurivillius structures for all the five samples with no secondary phase. Structures fitted from Rieveld refinements are shown in Table 1 with low $R_{\mathrm{wp}}$ obtained, indicating the correction of the fitness. As shown in Table 1, it can be clearly seen that $c$ parameters of samples vary largely and are about 25.1132, 29.1250, 33.4223, 37.3095, and 41.2454 ̊̊ for BTN-2, BLTNF-2.5, BLTNF-3, BLTNF3.5 , and BTF-4, respectively. The in-between layer length of BLTNF-2.5 and BLTNF-3.5, when compared with those of BTN2, BLTNF-3 and BTF-4, highly indicates the formation of intergrowth structure. Besides $c$ parameters, $a$ and $b$ parameters of BLTNF-2.5 and BLTNF-3.5 differ largely from their component oxides, which have integral layers, to alternately accommodate to the difference of two constituent lattices. Such accommodation can introduce an internal stress at the interface of the two constituent lattices like that in the superlattices, ${ }^{27-29}$ which may lead to additional lattice distortion, and therefore impact the characteristics of these compounds.

STEM-HAADF images of BLTNF-2.5, BLNTF-3 and BLTNF-3.5 are shown in Fig. 3 to reveal their atomic structure. Bright spots here represent $\mathrm{Bi}$ or La ions because their large atomic number in the compounds. For BLNTF-3, orderly structure with two loose layers (the perovskite layer) and two close layers of packing ions (fluorite layer) shows up, suggesting a well crystalline 3-layer Aurivillius structure. While for BLTNT-2.5, sandwiched by the two close layers, one and two loose layers with large layer distance arrange orderly and sequentially, indicating a 2-3 layer intergrowth structure. Similarly, a 3-4 layer intergrowth structure can be clearly observed for BLTNT-3.5.
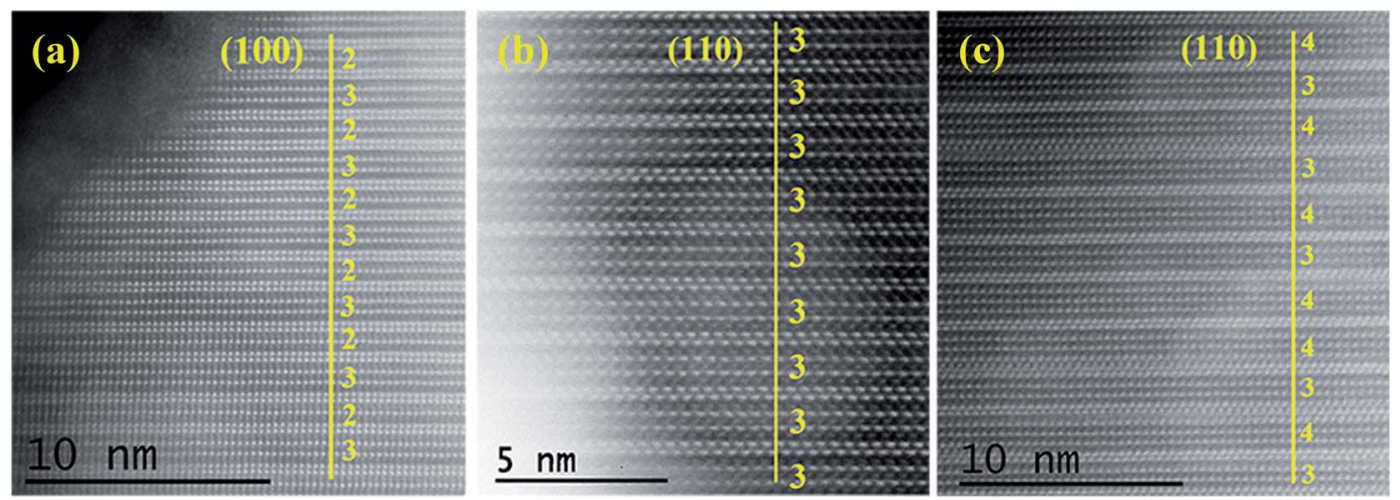

Fig. 3 STEM-HAADF images of (a) $\mathrm{Bi}_{3} \mathrm{TiNbO}_{9}-\mathrm{Bi}_{3} \mathrm{LaTiNbFeO}_{12}$ (BLTNF-2.5); (b) Bi $\mathrm{BLaTiNbFeO}_{12}\left(\mathrm{BLTNF}_{3}\right.$ ); and (c) $\mathrm{Bi}_{3} \mathrm{LaTiNbFeO}_{12}-\mathrm{Bi}_{5} \mathrm{Ti}_{3} \mathrm{FeO}_{15}$ (BLTNF-3.5). 


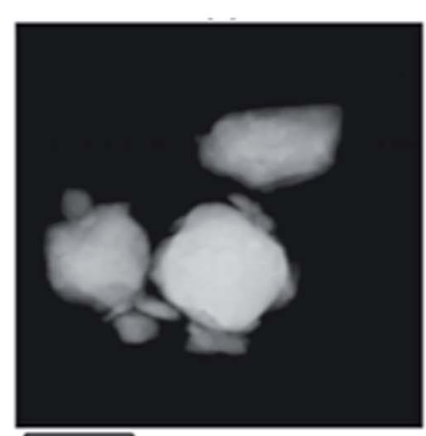

$250 \mathrm{~nm}$
Bi L $\alpha 1$

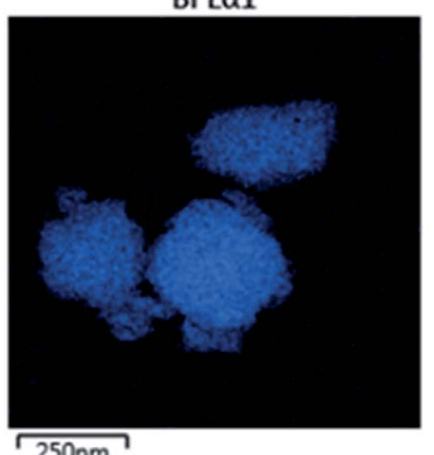

Fe $K \alpha 1$

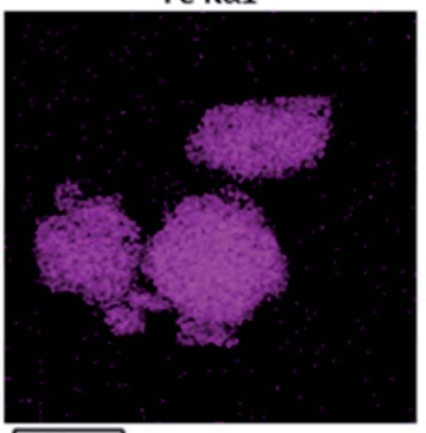

$\sqrt{250 \mathrm{~nm}}$

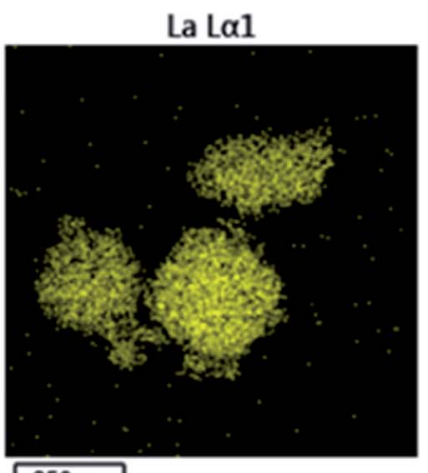

$\longdiv { 2 5 0 \mathrm { nm } }$
$\mathrm{Ti} K \alpha 1$

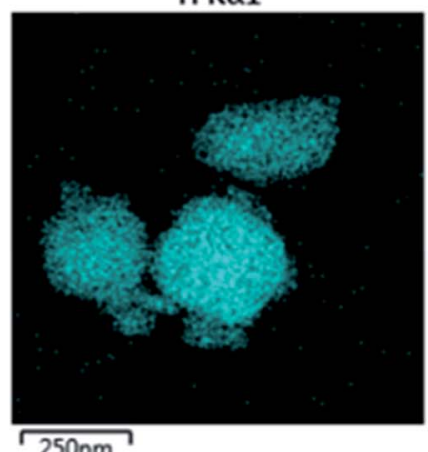

$\mathrm{O} K \alpha 1$

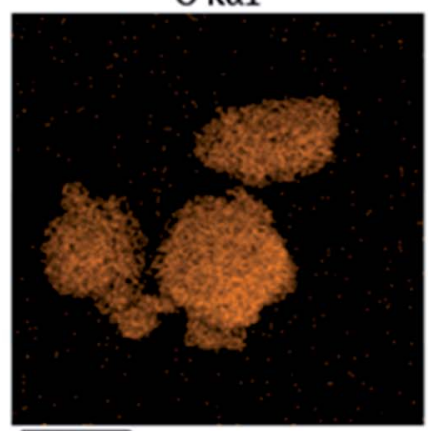

$\longdiv { 2 5 0 \mathrm { nm } }$

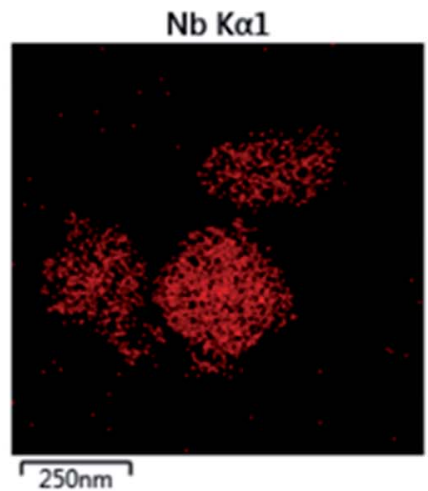

Fig. 4 TEM-EDS images of $\mathrm{Bi}_{3} \mathrm{LaTiNbFeO}{ }_{12}-\mathrm{Bi}_{5} \mathrm{Ti}_{3} \mathrm{FeO}_{15}$ (BLTNF-3.5).

Observation of these superlattice-like intergrowth structures intensively indicates the corrections of XRD refinements.

Fig. 4 shows TEM-EDS images of BLTNF-3.5. All the elements appear uniformly with no individual element enriched or lessened. This along with the XRD analysis further indicates that pure intergrowth samples can be prepared via a simple solid state reaction process.

Raman spectra, as shown in Fig. 5, are made to provide some valuable information about lattice properties and structural distortions. It is generally accepted that the Raman modes below $200 \mathrm{~cm}^{-1}$, are ascribed to the vibration of $\mathrm{Bi}^{3+}$ ions, while those above $200 \mathrm{~cm}^{-1}$ respond to the motion of $\mathrm{BO}_{6}(\mathrm{~B}=\mathrm{Ti}, \mathrm{Nb}$, $\mathrm{Fe}$ ) octahedral. ${ }^{30,31}$ Yet, restricted by low test limit of spectrometer, only incomplete peaks below $200 \mathrm{~cm}^{-1}$ are gotten in this work, and therefore, we focus on the shifting tendency of modes above $200 \mathrm{~cm}^{-1}$ with respect to the layer change in samples. It

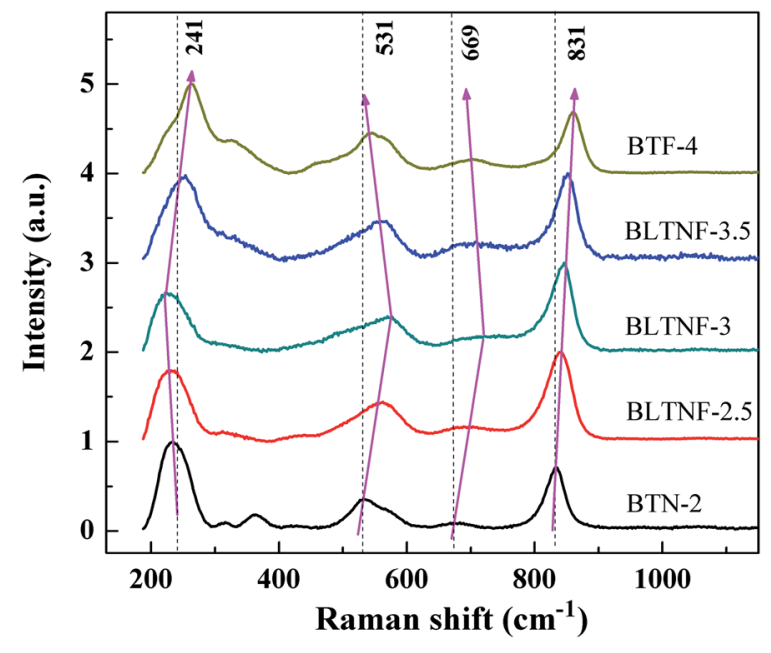

Fig. 5 Raman spectra of samples. 

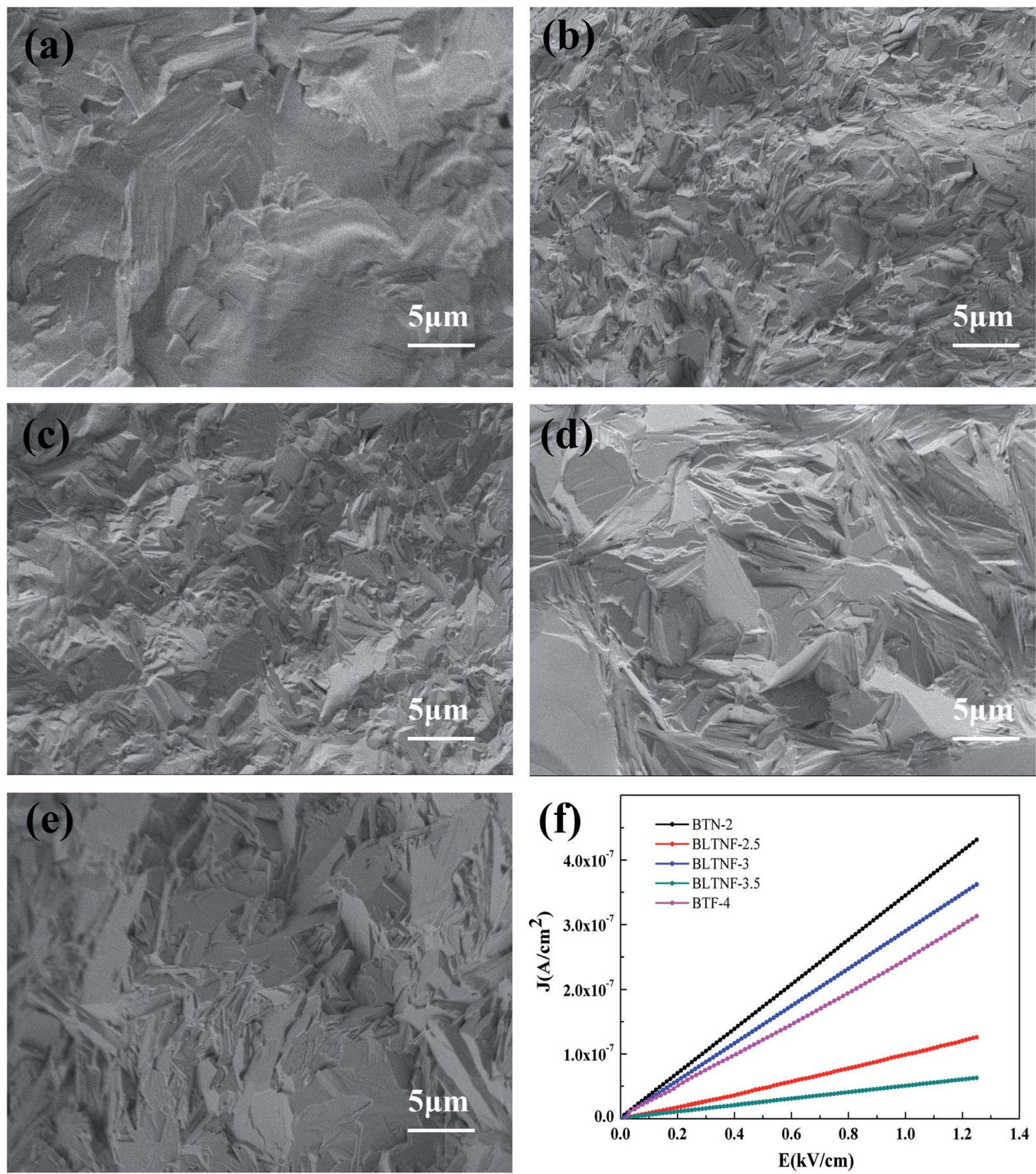

Fig. 6 SEM micrographs of (a) BTN-2; (b) BLTNF-2.5; (c) BLTNF-3; (d) BLTNF-3.5; (e) BTF-4; (f)The J-E curve of the samples.

has been reported that for BTN-2, the modes at $241 \mathrm{~cm}^{-1}$ represent the internal angle bending vibration of the $\mathrm{BO}_{6}$ octahedron; the modes at about $531 \mathrm{~cm}^{-1}$ correspond to the opposite excursions of the $\mathrm{O}$ atoms at the $\mathrm{BO}_{6}$ octahedron; the modes at about $669 \mathrm{~cm}^{-1}$ correspond to the band stretching of the $\mathrm{BO}_{6}$ octahedron; and the modes at about $831 \mathrm{~cm}^{-1}$ indicate the symmetric stretching of the $\mathrm{BO}_{6}(\mathrm{~B}=\mathrm{Ti}, \mathrm{Nb})$ octahedron..$^{30,32}$ With the increase of layer number, modes at $831 \mathrm{~cm}^{-1}$ shift steadily right, indicating its direct connection with the $c$ parameter. ${ }^{31}$ Interestingly, other modes show different shift tendency: modes at 531 and $669 \mathrm{~cm}^{-1}$ shift right first and then left; while those at $221 \mathrm{~cm}^{-1}$ shift left first and then right. Compared with the parameters listed in Table 1, these vibrations seem to show large positive or negative dependence on $a$ or $b$ parameters. This, in a large scale, indicates the existence of a superlattice-like structure, in good agreement with the STEM-HADDF observation. And further, the lattice distortion rooting in the superlattice-like structures can be expected. 

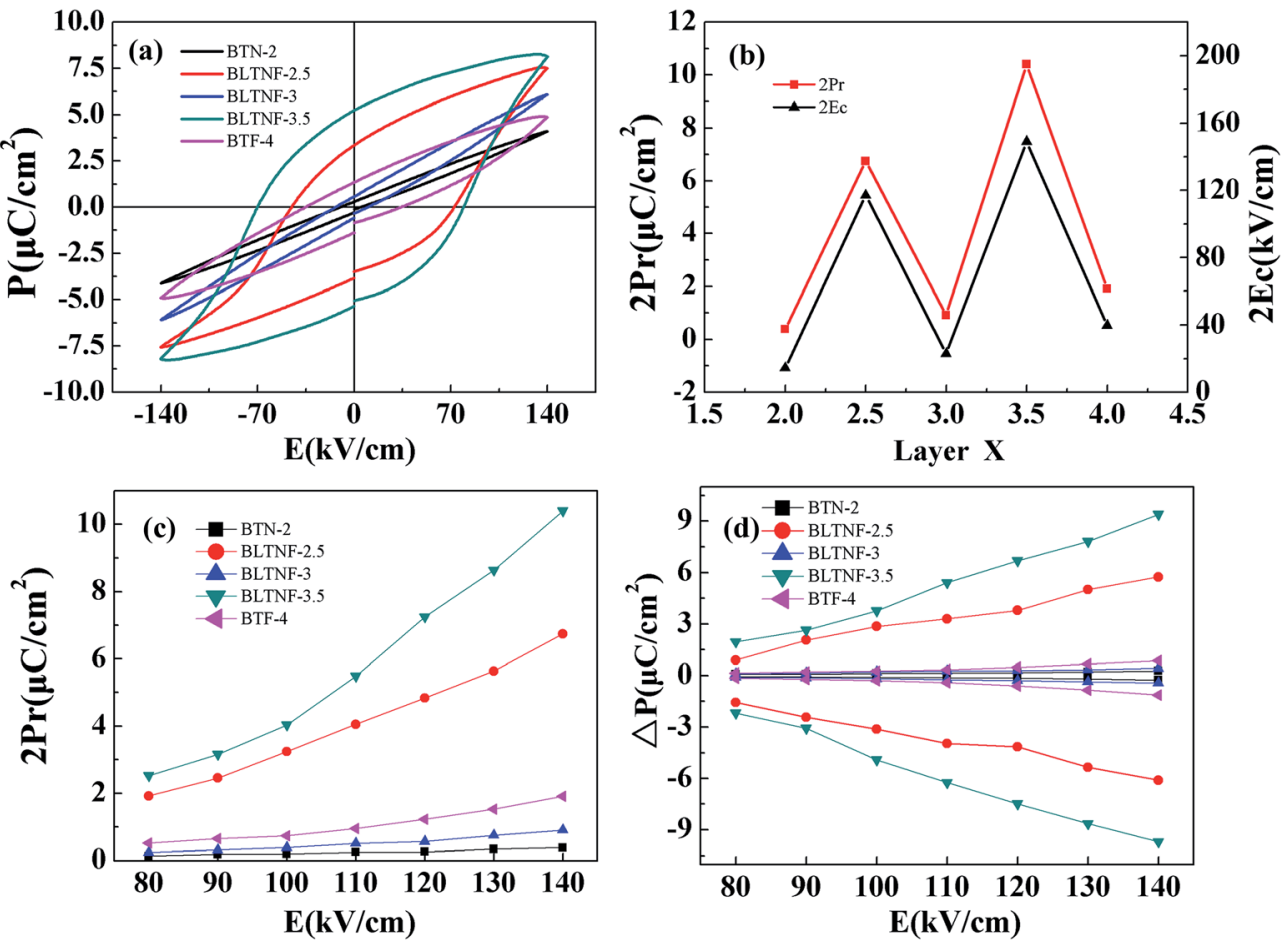

Fig. 7 (a) $P-E$ hysteresis loops of the samples at $140 \mathrm{kV} \mathrm{cm}^{-1}$ in $50 \mathrm{~Hz}$; (b) dependence of $2 E_{\mathrm{c}}$ and $2 P_{\mathrm{r}}$ on the number of layer $(x)$; and $2 P_{\mathrm{r}}$ (c) and $\Delta P(d)$ of the samples measured at different electrical field.

Before exploring the ferroelectric properties of the samples, SEM photos of fractured samples sintered at $1150{ }^{\circ} \mathrm{C}$ are taken as shown in Fig. 6 . All the five samples are very dense with few pores detected. High densities of these samples help to rule out the current leakage via pores, and thus benefit the identification of native FE properties. Conductive properties of the samples under the applied DC electric fields are investigated at the room temperature, as shown in Fig. 6(f). Based on the linear slope of $J-E$ curves, electrical resistivity of samples can be calculated as $2.8,10.1,3.4,15.4$, and $4.1 \mathrm{G} \Omega \mathrm{cm}$ for BTN, BLTNF-2.5, BLTNF3.5 and BTF-4, respectively. It can be clearly seen that BLTNF-3.5 and BLTNF-2.5 with the intergrowth structure have much higher electrical resistivity than those with integer-layer samples. This might result from the larger electron scattering because of the larger lattice distortion of the intergrowth structures.

The RT $P-E$ hysteresis loops measured at $50 \mathrm{~Hz}$ under different electric fields are shown in Fig. 7(a), and values of apparent remnant polarization $\left(2 P_{\mathrm{r}}\right)$ and coercive field $\left(2 E_{\mathrm{c}}\right)$ under the electric field $140 \mathrm{kV} \mathrm{cm}^{-1}$ are shown in Fig. 7(b) as function of layer numbers in these samples. Much enhanced $2 P_{\mathrm{r}}$ and $2 E_{\text {c }}$ values can be clearly observed for BLTNF-2.5 and BLTNF-3.5 with the values of 6.74 and $10.40 \mu \mathrm{C} \mathrm{cm}^{-2}$ and 117 , $149 \mathrm{kV} \mathrm{cm}^{-1}$, respectively. To identify a possible contribution from artificial polarization due to current leakage, pulsed polarization positive-up negative-down (PUND) measurements are performed, and the pulsed remnant polarizations $\Delta P$ (switched polarization-non-switched polarization) at different electric fields are shown in Fig. $7(\mathrm{~d})$. Values of $\Delta P$ are very close to those of $2 P_{\mathrm{r}}$ shown in Fig. $7(\mathrm{c})$, indicating that the polarization of the samples arises mainly from their intrinsic properties.

Reasons for the high intrinsic ferroelectric performance of samples with the intergrowth phases can be attributed to their superlattice-like structure. As shown in Table 1, parameters of the constituent sublattices differ a lot from each other, especially in $a$ and $b$ directions. Such mismatching in sublattices will introduce a large interfacial tension in the intergrowth structure, which can result in larger $\mathrm{BO}_{6}$ octahedron distortion and Jahn-Teller distortion, and thus improve the ferroelectric polarization.

Dielectric properties of the samples measured at various frequencies under temperature $373-923 \mathrm{~K}$ are conducted as shown in Fig. 8 to determine the ferroelectric Curie temperature. A bump shoulder at $610 \mathrm{~K}$ which shifts to high temperatures at the enhanced frequencies can be observed for BLTNF3.5. As indicated in literatures, such broad peak corresponds to the space charge polarization instead of Curie temperatures. ${ }^{33,34}$ Similar peaks are also observed for BTN-2 (849 K at 5 $\mathrm{KHz})$ and BTF-4 (766 K at $5 \mathrm{KHz})$. For BLTNF-3.5, the peak corresponding to Curie temperatures locates at $861 \mathrm{~K}$, which doesn't shift with the testing frequencies. Similarly, high Curie temperatures can be obtained as $780 \mathrm{~K}$ for BLTNF-2.5 and $875 \mathrm{~K}$ for BLTNF-3. For BFT-4 and BTN-2, their Curie temperatures are too high to be obtained here. ${ }^{35,36}$ Dielectric loss factor of samples is also plotted in Fig. 8. Although Curie temperatures can be obtained in dielectric loss curves (as indicated by the line), peaks corresponding to space charge are invisible. Whereas, additional peaks above Curie temperatures are 

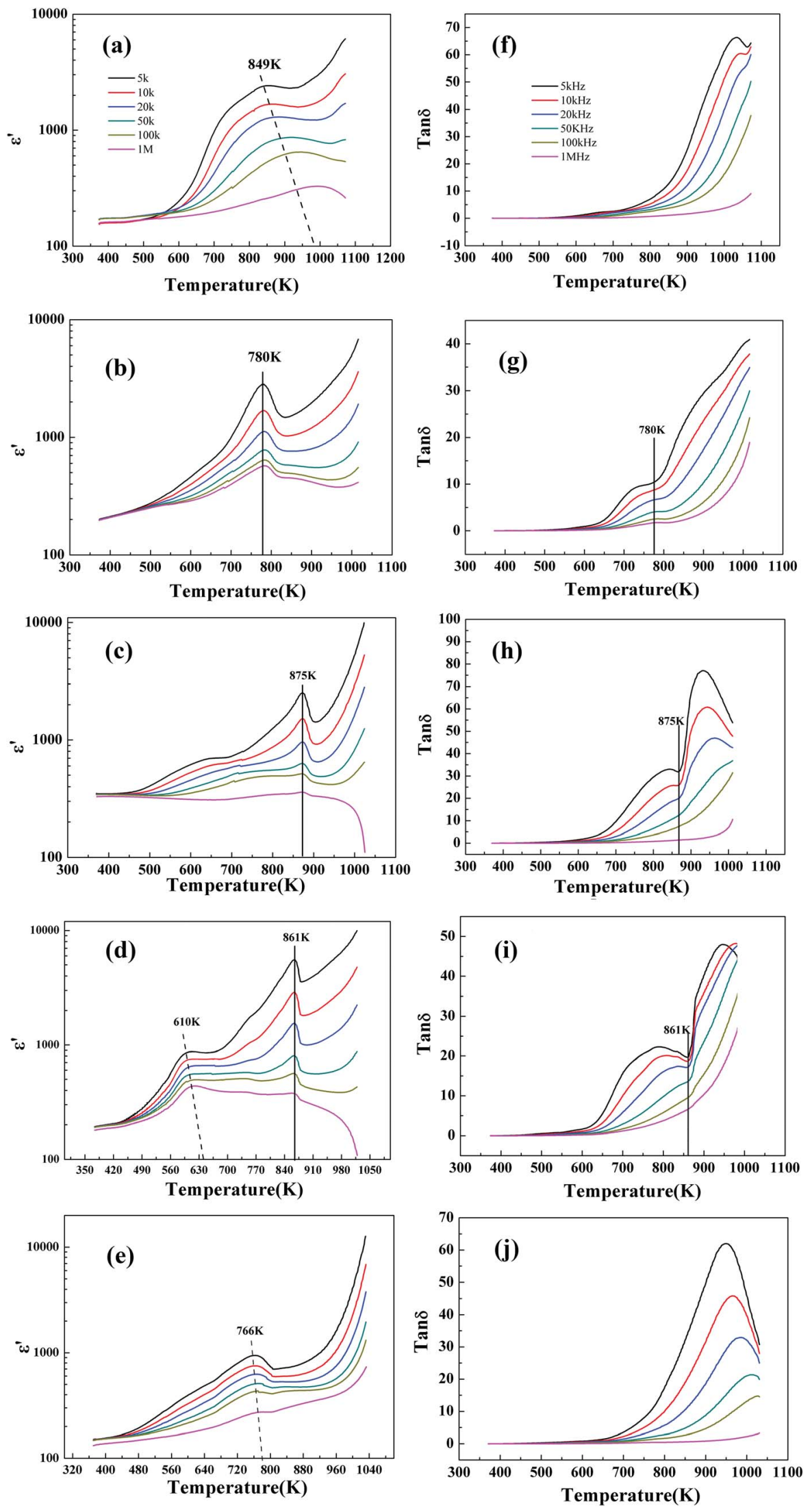

Fig. 8 Temperature dependency of dielectric constant $\left(\varepsilon^{\prime}\right)$ and dielectric loss factor (tan $\delta$ ) at $5 \mathrm{kHz}-1 \mathrm{MHz}$ of (a) and (f) BTN-2; (b) and (g) BLTNF2.5; (c) and (h) BLTNF-3; (d) and (i) BLTNF-3.5; and (e) and (j) BTF-4 samples. 

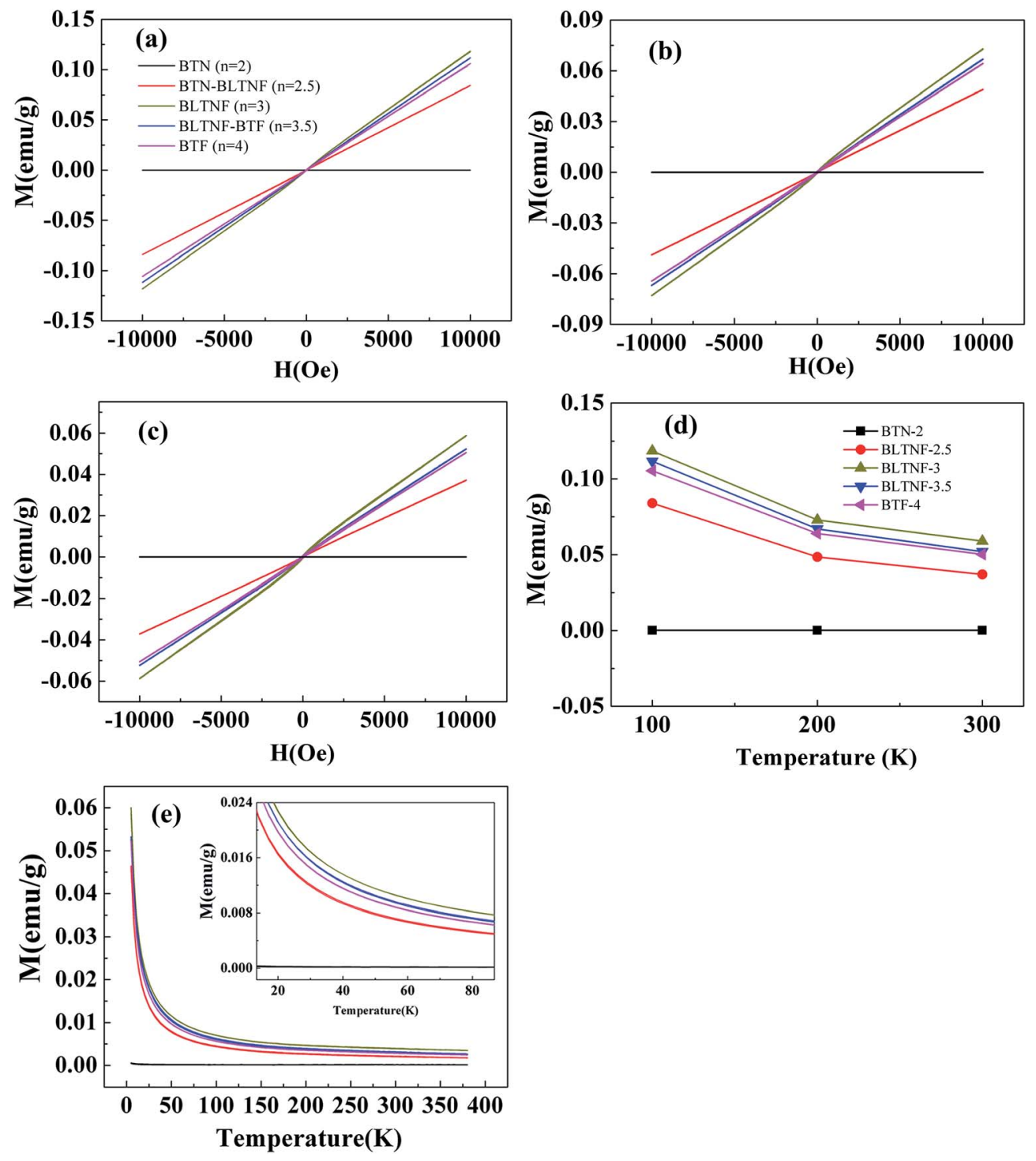

Fig. 9 The magnetic hysteresis loops of the samples measured at (a) $100 \mathrm{~K}$; (b) $200 \mathrm{~K}$; (c) $300 \mathrm{~K}$; (d) dependence of magnetic polarization measured at 10000 Oe and different temperatures; and (e) temperature dependence of magnetization of the samples in the ZFC-FC modes measured at 500 Oe.

Table 2 Iron mass fraction and effective magnetic moment $t$ for the samples

\begin{tabular}{lll}
\hline Compound & $\begin{array}{l}\text { Iron mass } \\
\text { fraction (\%) }\end{array}$ & $\begin{array}{l}\text { Effective magnetic } \\
\text { moment }\left(\mu_{\mathrm{B}}\right)\end{array}$ \\
\hline $\mathrm{Bi}_{3} \mathrm{TiNbO}_{9}$ & 0 & - \\
$\mathrm{Bi}_{3} \mathrm{TiNbO}_{9}-\mathrm{Bi}_{3} \mathrm{LaTiNbFeO}_{12}$ & 2.703 & 3.75 \\
$\mathrm{Bi}_{3} \mathrm{LaTiNbFeO}_{12}$ & 4.837 & 3.58 \\
$\mathrm{Bi}_{3} \mathrm{LaTiNbFeO}_{12}-\mathrm{Bi}_{5} \mathrm{Ti}_{3} \mathrm{FeO}_{15}$ & 4.233 & 3.46 \\
$\mathrm{Bi}_{5} \mathrm{Ti}_{3} \mathrm{FeO}_{15}$ & 3.888 & 3.83 \\
\hline
\end{tabular}

observed in BTN-2, BLTNF-3, BLTNF-3.5 and BTF-4 samples, corresponding to dielectric relaxation. ${ }^{33}$

It should be also noted that the samples with intergrowth structures have lower Curie temperatures when compared with those having their component lattices. Such low Curie temperature should result from the internal stress of the superlattice-like structure which lowers the phase transformation energy.

At last, we conducted a magnetic test to investigate the magnet performance. Fig. 9 shows the magnetic hysteresis loops of the samples measured at different temperatures and the temperature dependence of magnetization for the samples in zero field cooling (ZFC) and field cooling (FC) modes under a magnetic field of 500 Oe. BTN-2 shows diamagnetism character, while other samples are paramagnetic which increases with the $\mathrm{Fe}^{3+}$ contents. As indicated in Fig. 9(d), paramagnetism of samples decreases with increasing temperatures due to the disarranging of magnetic domain at high temperatures. Effective magnetic moments $\left(M_{\text {eff }}\right)$ of iron are calculated for each samples using the Curie-Weiss law. ${ }^{37}$ As shown in Table 2, BLTNF-2.5, BLTNF-3, BLTNF-3.5 and BFT-4 have similar 
effective magnetic moments around 3.45-3.85. As all known that the theoretical effective magnetic moment for iron can be calculated as $3.46 \mu_{\mathrm{B}}$ at low spin state and $11.83 \mu_{\mathrm{B}}$ at high spin state. And therefore, the iron ions in these samples are almost all at the low spin state. The slight difference here may be due to different environment of the $\mathrm{FeO}_{6}$ octahedral which has slight different crystal field splitting energy according to Jahn-Taller effect. It is also suggested that pure paramagnetic in these samples are mainly dependent on their iron contents, while hardly on their homogeneous or heterogeneous structure (intergrowth structure).

\section{Conclusions}

In summary, the intergrowth Aurivillius-type multiferroic oxide BLTNF-2.5 and BLTNF-3.5 were successfully synthesized via solid-state reaction method. The XRD and STEM-HADDF results proved that these oxides have a superlattice-like structure compared with their constituent oxides BTN-2, BLTNF-3 and BTF-4. Moreover, ferroelectric polarization and coercive filed of BLTNF-2.5 and BLTNF-3.5 had been greatly improved with the lower current leakage which should be attributed to their superlattice-like structure. Yet, magnetic measurements suggest that BLTNF-2.5 and BLTNF-3.5 present paramagnetic properties possessing similar effective magnetic moment with $\mathrm{Bi}_{3} \mathrm{LaTiNbFeO}_{12}$ and $\mathrm{Bi}_{5} \mathrm{Ti}_{3} \mathrm{FeO}_{15}$, implying that such superlattice-like structure seems to have less impact on their magnetic properties. These results might provide a new perspective for the development of the multiferroic family.

\section{Conflicts of interest}

There are no conflicts to declare.

\section{Acknowledgements}

This work was supported by the National Key R\&D Program of China (2016YFA0401004), the Chinese Universities Scientific Fund (CUSF, WK2310000055), Anhui Provincial Natural Science Foundation (P. R. China, No. 1608085QE91), and the External Cooperation Program of BIC (Chinese Academy of Sciences, No. 211134KYSB20130017).

\section{Notes and references}

1 Y. Moritomo, A. Asamitsu, H. Kuwahara and Y. Tokura, Giant magnetoresistance of manganese oxides with a layered perovskite structure, Nature, 1996, 380, 141-144.

2 P. Mandal, M. Pitcher, J. Alaria, H. Niu, P. Borisov, P. Stamenov, J. Claridge and M. Rosseinsky, Designing switchable polarization and magnetization at room temperature in an oxide, Nature, 2015, 525, 363-366.

3 M. Fiebig, Revival of the magnetoelectric effect, J. Phys. D: Appl. Phys., 2005, 38, R123.

4 A. C. Garcia-Castro, A. H. Romero and E. Bousquet, StrainEngineered Multiferroicity in Pnma $\mathrm{NaMnF}_{3}$ Fluoroperovskite, Phys. Rev. Lett., 2016, 116, 117202.
5 S. Ikegami and I. Ueda, Piezoelectricity in ceramics of ferroelectric bismuth compound with layer structure, Jpn. J. Appl. Phys., 1974, 13, 1572.

6 A. Yadav, C. Nelson, S. Hsu, Z. Hong, J. Clarkson, C. Schlepütz, A. Damodaran, P. Shafer, E. Arenholz and L. Dedon, Observation of polar vortices in oxide superlattices, Nature, 2016, 530, 198-201.

7 S. Zhang and F. Yu, Piezoelectric materials for high temperature sensors, J. Am. Ceram. Soc., 2011, 94, 3153-3170.

8 R. Singh, T. Bhimasankaram, G. Kumar and S. Suryanarayana, Dielectric and magnetoelectric properties of $\mathrm{Bi}_{5} \mathrm{FeTi}_{3} \mathrm{O}_{15}$, Solid State Commun., 1994, 91, 567-569.

9 M. Krzhizhanovskaya, S. Filatov, V. Gusarov, P. Paufler, R. Bubnova, M. Morozov and D. Meyer, Aurivillius phases in the $\mathrm{Bi}_{4} \mathrm{Ti}_{3} \mathrm{O}_{12} / \mathrm{BiFeO}_{3}$ system: Thermal behaviour and crystal structure, Z. Anorg. Allg. Chem., 2005, 631, 1603-1608.

10 X. Mao, W. Wang, X. Chen and Y. Lu, Multiferroic properties of layer-structured $\mathrm{Bi}_{5} \mathrm{Fe}_{0.5} \mathrm{Co}_{0.5} \mathrm{Ti}_{3} \mathrm{O}_{15}$ ceramics, Appl. Phys. Lett., 2009, 95, 082901.

11 J. Wang, Z. Fu, R. Peng, M. Liu, S. Sun, H. Huang, L. Li, R. J. Knize and Y. Lu, Low magnetic field response singlephase multiferroics under high temperature, Mater. Horiz., 2015, 2, 232-236.

12 X. Li, Z. Ju, F. Li, Y. Huang, Y. Xie, Z. Fu, R. Knize and Y. Lu, Visible light responsive $\mathrm{Bi}_{7} \mathrm{Fe}_{3} \mathrm{Ti}_{3} \mathrm{O}_{21}$ nanoshelf photocatalysts with ferroelectricity and ferromagnetism, $J$. Mater. Chem. A, 2014, 2, 13366-13372.

13 B. Aurivillius, Mixed Bismuth Oxides with Layer lattices I. The structure type of $\mathrm{CaNb}_{2} \mathrm{Bi}_{2} \mathrm{O}_{9}$, Ark. Kemi, 1949, 1, 463480.

14 S. Sun, Y. Huang, G. Wang, J. Wang, Z. Fu, R. Peng, R. J. Knize and Y. Lu, Nanoscale structural modulation and enhanced room-temperature multiferroic properties, Nanoscale, 2014, 6, 13494-13500.

15 G. Wang, H. Yang, J. Wang, S. Sun, Z. Fu, X. Zhai, R. Peng, R. J. Knize and Y. Lu, Engineering the exchange bias and bias temperature by modulating the spin glassy state in single phase $\mathrm{Bi}_{9} \mathrm{Fe}_{5} \mathrm{Ti}_{3} \mathrm{O}_{27}$, Nanoscale, 2017, 9, 8305-8313.

16 J. Zhu, W.-P. Lu, X.-Y. Mao, R. Hui and X.-B. Chen, Study on properties of lanthanum doped $\mathrm{SrBi}_{4} \mathrm{Ti}_{4} \mathrm{O}_{15}$ and $\mathrm{Sr}_{2} \mathrm{Bi}_{4} \mathrm{Ti}_{5} \mathrm{O}_{18}$ ferroelectric ceramics, Jpn. J. Appl. Phys., 2003, 42, 5165.

17 D. Mercurio, G. Trolliard, T. Hansen and J. Mercurio, Crystal structure of the ferroelectric mixed Aurivillius phase $\mathrm{Bi}_{7} \mathrm{Ti}_{4} \mathrm{NbO}_{21}$, Int. J. Inorg. Mater., 2000, 2, 397-406.

18 Z. Yi, Y. Wang, Y. Li and Q. Yin, Ferroelectricity in intergrowth $\mathrm{Bi}_{3} \mathrm{TiNbO}_{9}-\mathrm{Bi}_{4} \mathrm{Ti}_{3} \mathrm{O}_{12}$ ceramics, J. Appl. Phys., 2006, 99, 114101.

19 Z. Yi, Y. Li, Z. Wen, S. Wang, J. Zeng and Q. Yin, Intergrowth $\mathrm{Bi}_{2} \mathrm{WO}_{6}-\mathrm{Bi}_{3} \mathrm{TiNbO}_{9}$ ferroelectrics with high ionic conductivity, Appl. Phys. Lett., 2005, 86, 192906.

20 I. Ismailzade, V. Nesterenko, F. Mirishli and P. Rustamov, XRay and electrical studies of system $\mathrm{Bi}_{4} \mathrm{Ti}_{3} \mathrm{O}_{12}-\mathrm{BiFeO}_{3}$, Soviet Physics - Crystallography, 1967, 12, 400-405.

21 S. Nakashima, H. Fujisawa, S. Ichikawa, J. M. Park, T. Kanashima, M. Okuyama and M. Shimizu, Structural and ferroelectric properties of epitaxial $\mathrm{Bi}_{5} \mathrm{Ti}_{3} \mathrm{FeO}_{15}$ and 
natural-superlattice-structured $\mathrm{Bi}_{4} \mathrm{Ti}_{3} \mathrm{O}_{2}-\mathrm{Bi}_{5} \mathrm{Ti}_{3} \mathrm{FeO}_{15}$ thin films, J. Appl. Phys., 2010, 108, 074106.

22 X. Gao, H. Gu, Y.-X. Li, Z.-G. Yi, M. Čeh and K. Žagar, Structural evolution of the intergrowth bismuth-layered $\mathrm{Bi}_{7} \mathrm{Ti}_{4} \mathrm{NbO}_{21}$, J. Mater. Sci., 2011, 46, 5423-5431.

23 H. Ogawa, D. Iida, S. Takahashi, T. Moriyama and A. Kan, Ferroelectric properties and crystal structure of mixed-layer $\mathrm{Bi}_{7-\mathrm{x}} \mathrm{Sr}_{\mathrm{x}} \mathrm{Ti}_{4} \mathrm{Nb}_{1-\mathrm{x}} \mathrm{W}_{\mathrm{x}} \mathrm{O}_{21}$ ceramics, Ferroelectrics, 2016, 498, 111.

24 X. Tian, S. Qu, B. Wang, H. Du and Z. Xu, Intergrowth Bismuth Layer-Structured $\mathrm{Na}_{0.5} \mathrm{Bi}_{2.5} \mathrm{Nb}_{2} \mathrm{O}_{9}-\mathrm{Bi}_{4} \mathrm{Ti}_{3} \mathrm{O}_{12}$ High Temperature Ferroelectrics Ceramics, J. Inorg. Organomet. Polym. Mater., 2014, 24, 355-359.

25 H. Zou, J. Li, Q. Cao, X. Wang, X. Hui, Y. Li, Y. Yu and X. Yao, Intensive up-conversion photoluminescence of $\mathrm{Er}^{3+}$-doped $\mathrm{Bi}_{7} \mathrm{Ti}_{4} \mathrm{NbO}_{21}$ ferroelectric ceramics and its temperature sensing, J. Adv. Dielectr., 2014, 4, 1450028.

26 H. Zhang, H. Yan and M. J. Reece, High temperature leadfree relaxor ferroelectric: Intergrowth Aurivillius phase $\mathrm{BaBi}_{2} \mathrm{Nb}_{2} \mathrm{O}_{9}-\mathrm{Bi}_{4} \mathrm{Ti}_{3} \mathrm{O}_{12}$ ceramics, J. Appl. Phys., 2010, 107, 104111.

27 K. J. Choi, M. Biegalski, Y. Li, A. Sharan, J. Schubert, R. Uecker, P. Reiche, Y. Chen, X. Pan and V. Gopalan, Enhancement of ferroelectricity in strained $\mathrm{BaTiO}_{3}$ thin films, Science, 2004, 306, 1005-1009.

$28 \mathrm{~J}$. Junquera and P. Ghosez, Critical thickness for ferroelectricity in perovskite ultrathin films, Nature, 2003, 422, 506-509.

29 L. W. Martin and A. M. Rappe, Thin-film ferroelectric materials and their applications, Nat. Rev. Mater., 2016, 2, 16087.
30 S. Kojima, R. Imaizumi, S. Hamazaki and M. Takashige, Raman scattering study of bismuth layer-structure ferroelectrics, Jpn. J. Appl. Phys., 1994, 33, 5559.

31 P. Graves, G. Hua, S. Myhra and J. Thompson, The Raman modes of the Aurivillius phases: temperature and polarization dependence, J. Solid State Chem., 1995, 114, 112-122.

32 Y. Du, M. Zhang, Q. Chen, Z. Yuan, Z. Yin and Q. Zhang, Size effect and evidence of a size-driven phase transition in $\mathrm{Bi}_{4} \mathrm{Ti}_{3} \mathrm{O}_{12}$ nanocrystals, Solid State Commun., 2002, 124, 113-118.

33 J. Yang, L. Yin, Z. Liu, X. Zhu, W. Song, J. Dai, Z. Yang and Y. Sun, Magnetic and dielectric properties of Aurivillius phase $\mathrm{Bi}_{6} \mathrm{Fe}_{2} \mathrm{Ti}_{3} \mathrm{O}_{18}$ and the doped compounds, Appl. Phys. Lett., 2012, 101, 012402.

34 A. Srinivas, M. M. Kumar, S. Suryanarayana and T. Bhimasankaram, Investigation of dielectric and magnetic nature of $\mathrm{Bi}_{7} \mathrm{Fe}_{3} \mathrm{Ti}_{3} \mathrm{O}_{21}$, Mater. Res. Bull., 1999, 34, 989-996.

35 A. Moure and L. Pardo, Microstructure and texture dependence of the dielectric anomalies and dc conductivity of $\mathrm{Bi}_{3} \mathrm{TiNbO}_{9}$ ferroelectric ceramics, J. Appl. Phys., 2005, 97, 084103.

36 X. Mao, W. Wang, H. Sun, Y. Lu and X. Chen, Influence of different synthesizing steps on the multiferroic properties of $\mathrm{Bi}_{5} \mathrm{Fe}_{1} \mathrm{Ti}_{3} \mathrm{O}_{15}$ and $\mathrm{Bi}_{5} \mathrm{Fe}_{0.5} \mathrm{Co}_{0.5} \mathrm{Ti}_{3} \mathrm{O}_{15}$ ceramics, J. Mater. Sci., 2012, 47, 2960-2965.

37 X. Zuo, M. Zhang, E. He, B. Guan, Y. Qin, J. Yang, X. Zhu and J. Dai, Structural, magnetic, and dielectric properties of W/ Cr co-substituted Aurivillius $\mathrm{Bi}_{5} \mathrm{FeTi}_{3} \mathrm{O}_{15}$, J. Alloys Compd., 2017, 726, 1040-1046. 